\title{
TREATMENT OF BIRTH FRACTURES AT FORDHAM HOSPITAL *
}

\author{
SAMUEL W. BOORSTEIN, M.D. \\ NEW YORK
}

That fractures do occur during delivery is an established fact. Fortunately, they are not frequent, but these cases cause great anxiety to the physician as well as to the parents.

These fractures are true fractures and not green stick fractures as was believed to be the case, hence deformity must be watched for and guarded against. Truesdell ${ }^{1}$ has proven this by his studies.

The first problem is how to treat these children. The methods used in the treatment of fractures occurring in adults are not adaptable for infants. Many surgeons have used different devices for the treatment of birth fractures.

For cases of fracture of the femur Scudder ${ }^{2}$ advises to place the leg in a flexed position on the body similar to the fetal position, the front of the thigh resting on the front of the abdomen, the foot reaching to the shoulder. The trunk is protected by powder and a folded soft towel so that there will be no chafing between the thigh or leg and the body. The position of the lower extremity is maintained by a swathe carefully adjusted or by turns of a bandage. This position is borne well by the child and is maintained for about three weeks. Each day the swathe is removed, the position being maintained while the parts are powdered, the towel readjusted and the leg massaged. Stimson recommended vertical extension of both legs.

Eisendrath ${ }^{3}$ says: "In children a fracture of the shaft is most easily treated by the use of Schede's vertical suspension."

The Buck and Hodgen splints are not well adapted for use in children.

Speed ${ }^{4}$ advises suspension of both legs at a right angle to the body. The legs are held by Buck's extension attached to a rope and pulley inserted in a longitudinal bar erected over the crib. Enough weight is applied to lift the buttock slightly from the bed surface.

Silver ${ }^{5}$ has combined the use of the Bradford frame and extension in a very useful manner. At a point opposite the hip joint, when the

* From the First Surgical Division, Dr. Alexander Nicoll, Director.

* Read in part before Bronx County Medical Society, May 21, 1919.

1. Truesdell, E. D.: Birth Fractures and Epiphyseal Dislocations, Hoe, 1917.

2. Scudder, C. L.: Treatment of Fractures, Ed. 8, Philadelphia, W. B. Saunders Co., 1918.

3. Keen's Surgery.

4. Speed, K.: Fractures and Dislocations, Philadelphia, Lea \& Febiger, 1919.

5. Silver, D.: Surg., Gynec. \& Obst., May, 1914, p. 645. 
child lies inside the frame, a $T$ connection is tightly screwed into the pipe. To this connection is attached a piece of pipe long enough to reach about four inches above the suspended foot. An L connection, capped at the end, furnishes the termination for this projection. The canvas covering the frame is made from one piece, a hole being cut

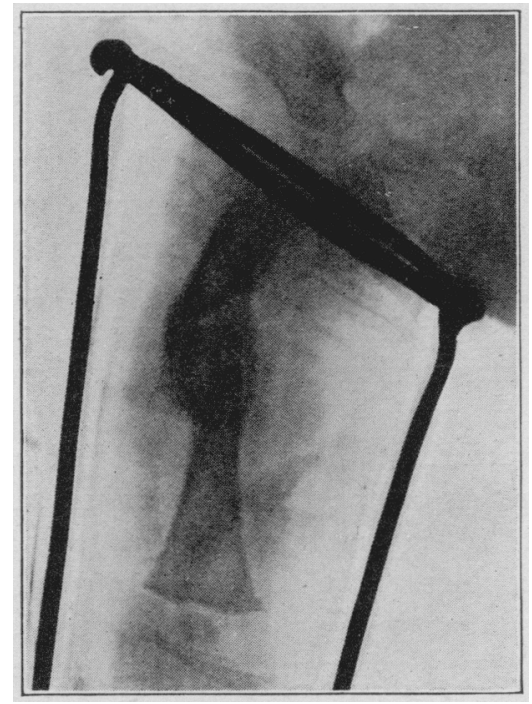

Fig. 1.-Fracture of femur. Splint in place, with marked callus present.

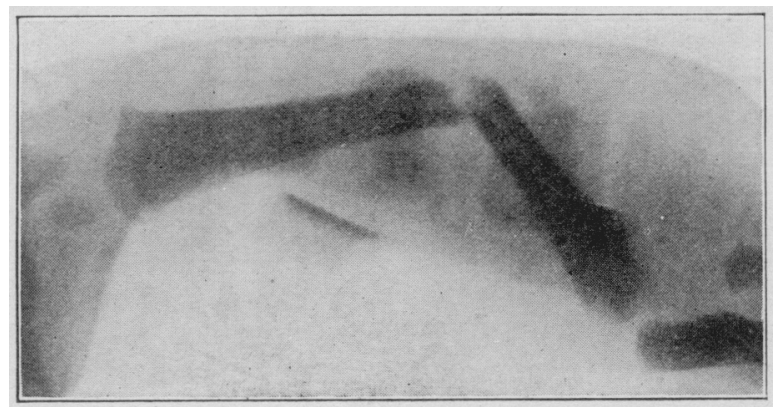

Fig. 2.-Fracture of humerus, before application of splint.

for the passage of the upright described. If the surgeon desires to suspend both legs, the frame can be shortened, as it does not have to care for one leg lying extended, and the terminal piece of the upright is longer. Adhesive plaster is used. The leg can be steadied by carrying the diaper around the upright piece or an adhesive strapping can be applied around the groin and the base of the upright. 
Russel and Reynolds, ${ }^{6}$ trying to avoid the use of adhesive plaster on the delicate skin of infants, have used the following method for a case of fracture of the femur:

"A plaster saddle was molded to fit the under surface of the knee which was reflexed to form an angle of about 120 degrees with the thigh, and was carried below the foot about $31 / 2$ inches to act as a counterweight; this saddle, suitably padded, was bandaged on beneath the knee and suspended from the crossbar of an apparatus constructed

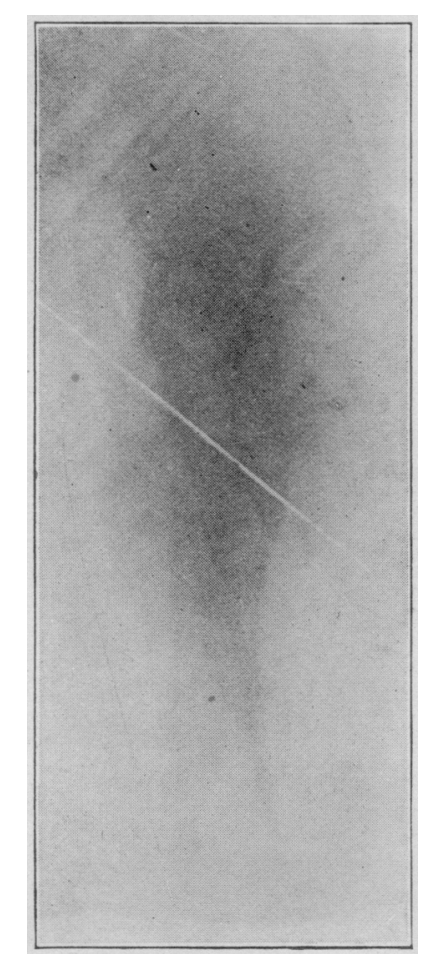

Fig. 3.-Fracture of femur. Good position of femur and much callus.

of a board 13 by 32 inches with side strips 3 inches wide, and with a 21 -inch upright at each end, to which was attached a horizontal crosspiece, the suspension being carried out by means of adhesive straps around the saddle, and a cord and pulleys and a 3-pound weight. The

- extension of plaster below the foot acted as a counterweight to hold the foot down and prevent the saddle from sliding out of position. As there was a slight tendency for the limb to swing from its proper axis after the patient's toilet, etc., had been attended to, a string was attached to the end of the saddle and the latter held in proper position

6. Russel, T. H., and Reynolds, H. S.: Birth Fractures of Femur, J. A. M. A. 68: 1902 (June 23) 1917. 
with slight traction by means of a light weight attached to one of the pulleys. When the limb was suspended the patient promptly fell asleep, evidencing the comfort of the apparatus."

Truesdell ${ }^{1}$ devised a box splint for the treatment of birth fractures of the femur.

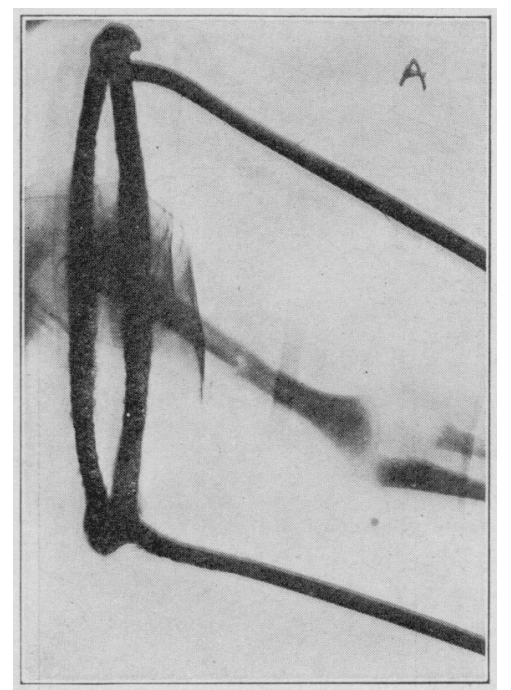

Fig. 4.-Proper adjustment of the splint to the fractured humerus.

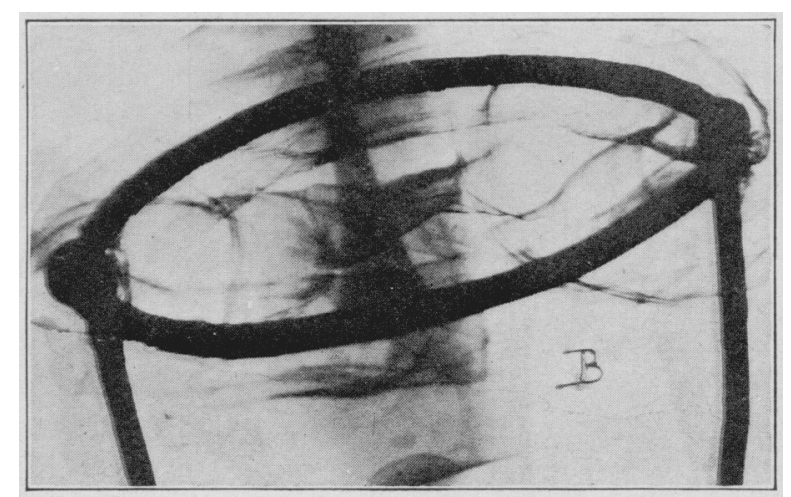

Fig. 5.-Improper adjustment of splint to the fractured femur. This was corrected afterward.

"The splint is built on a base board sixteen inches long, five and one-half inches wide and one inch thick. The sideboards are five and one-half inches high, and cut away for five inches to a height of three inches at the lower end of the box to allow for the band passing over the infant's abdomen to afford countertraction. The upright part of 
the splint is sixteen inches long, by two inches wide and one-half inch thick. The upright has an inclination of from ten to fifteen degrees, to bring the flexion of the injured thigh slightly beyond a right angle with the body. It is also inclined sideways to a corresponding degree, to afford abduction of the leg. The inner side of the upright arises from the center of the base board, while the outer side is shaped below to meet the end of the corresponding side-board, thus to strengthen its position. One large or two smaller pulleys are fixed to the upper end of the upright to carry the traction cord, while a screw hook at the base is used to fasten the large elastic band by which the traction is made."
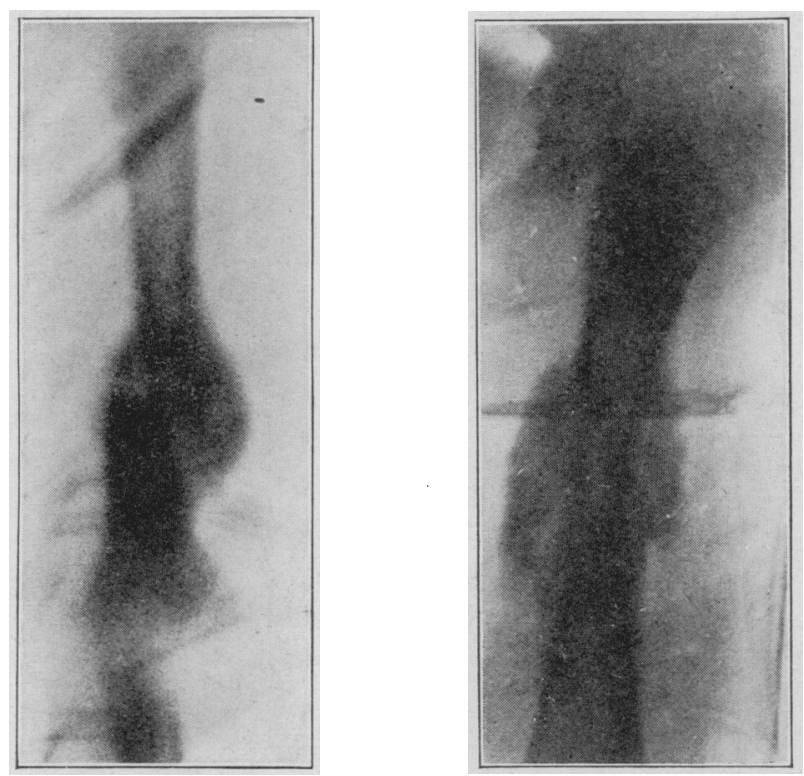

Fig. 6.-Final result in both the fractured humerus and the femur.

All the methods enumerated necessitate keeping the infant constantly in bed and under constant supervision. Scudder's method is, perhaps, easier to carry out, but it also requires a great deal of intelligence on the part of the mother.

On the first surgical division of Fordham Hospital, the surgical director, Dr. Alexander Nicoll, was kind enough to give me the opportunity of treating all the cases of birth fracture. These cases, together with the cases in my clinic in the out-patient department or in private practice, give me an opportunity to report the results obtained.

A new method, or to be more correct, the method introduced during the war for the treatment of fracture of the femur or hand in the adult, was used in all cases. We found it very satisfactory, and in all 
the cases in which it was used the method appeared to be quite superior to the other forms of apparatus. We feel justified to advocate its use, so that more extensive studies could be done in that line.

We made use of the Thomas Jones' splint. The method of application is so well known that it needs only a few lines of directions. ${ }^{7}$

The directions are: A strip of adhesive plaster to which a strong loop has been sewn at one end, is applied to each side of the leg or arm. The adhesive plasters should reach from as near the lesion as possible to the malleoli or to the wrist. The lower end of the strip should extend at least 6 inches below the sole of the foot or 4 inches

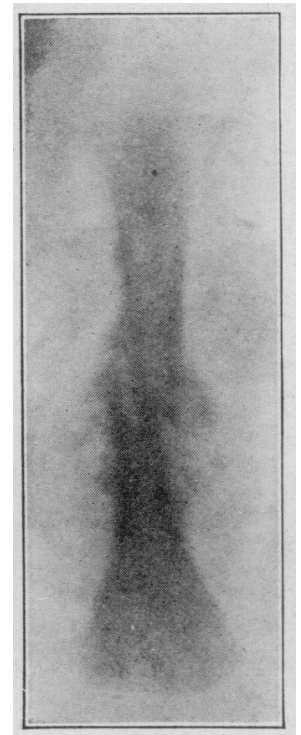

Fig. 7.-Fracture of humerus; good position of fragments.

beyond the tips of the fingers, and the sticky surfaces are covered with a piece of bandage so that they will not adhere. These ends are now tied to pieces of stout bandage which, in turn, are passed the one over and the other under the wire rods of the splint and returning to the center of the splint they are wound in the opposite direction once around the indentation or notch at the lower end of the splint and are then tied. A small piece of wood, in length about the width of the lower end of the splint, is now inserted an inch or two between the extension bands about 2 inches from the end of the splint, and the amount of traction is increased to any extent by twisting after the manner of a Spanish windlass or the old fashioned link-saw rope.

7. Any book on military orthopedics, fracture or surgery has the directions. 
This is retained by catching the end of the wood under or over one of the rods of the splint as the twist requires.

Instead of adhesive plaster we used moleskin plaster, or Sinclair's glue, which does not irritate the skin. The moleskin plaster is so often used in orthopedic practice in treating tubercular joints in children and as it is left on for months without causing irritation, there is no reason why we should hesitate to apply it for a few weeks, even though the patient is an infant.

The peroneal ring is not as tightly applied as in adults, and it is covered with flannel or chamois. The brace extends about 3 or 4 inches beyond the foot. The flannel covering the ring can easily be slipped out and changed every two or three days and the skin be well powdered. To prevent drop foot, a tongue depressor can be placed on the plantar

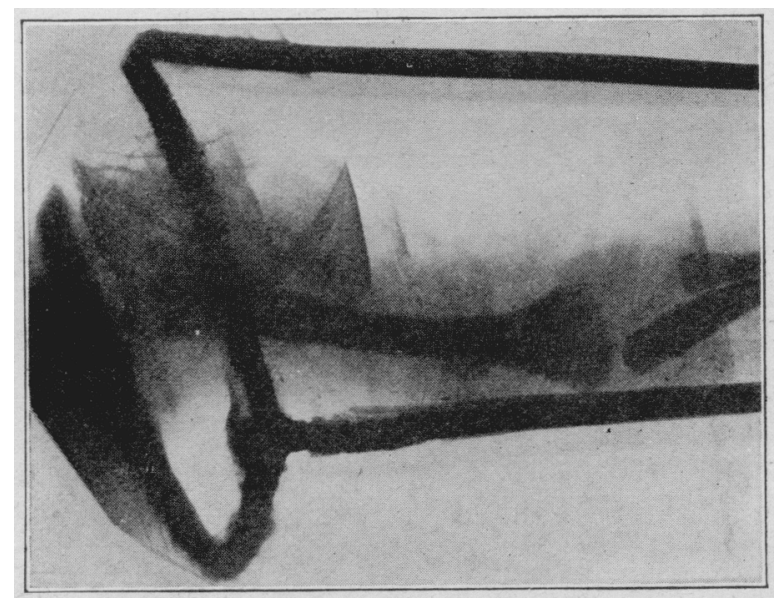

Fig. 8.-Final result; good union and good position.

surface of the foot and held by bandages to the bars of the splint. While wearing the brace, the child can easily be carried around, which means a great deal to the happy mother who is anxious to show the child to the admiring relatives, or to the mother who is burdened with some other important house duties.

A roentgenogram can easily be taken and the results watched. It is also easy to administer massage through the flannel or muslin bandage. The child lifts up the foot together with the brace, and thus the proper voluntary movements are made and union is hastened.

For the upper extremity, the ring is applied to the same shoulder or to the opposite shoulder (the good one). The braces are left on for from three to five weeks. Only two precautions are necessary: (1) The foot or the hand has to be watched for swelling. That can 
be prevented if the bands are loosened up once in a while and massaged. (2) The ring must not be too wide, as it will press on the axillary vessels, if the case is one involving the upper extremity, or on the other thigh if the case is one involving the lower extremity, and thus produce a deformity.

The children treated thus far have all done well, both the hospital cases and the ambulatory cases.

$I$ will report a few cases in detail.

\section{REPORT OF CASES}

CASE 1.-V. V., one of twins. This was the sixth pregnancy of a poor Italian mother. The delivery was made by a midwife. The infant sustained a fracture of the left femur. It was improperly treated for four weeks. I found no union present, with overlapping of the fragments (Fig. 1). A Thomas Jones splint was applied for two weeks. The length of the leg was increased, but there was still prominence of the upper fragment anteriorly. Under anesthesia the malposition was corrected and the splint reapplied. Massage was resorted to one week later. After three weeks union was good and position good. The child is now $2 \frac{1}{2}$ years old and there is no shortening of the limb.

CASE 2.-D. V., fracture of the left femur. The child was brought to the hospital when 4 weeks old. A splint was applied. Massage began one week later. In four weeks she was discharged as cured.

CASE 3. - Becky S., was delivered at the hospital with a fractured right humerus. She was referred to me by Dr. J. Telfair, the attending obstetrician of the hospital. The fracture was at the middle third of the humerus and the upper fragment was displaced upward. A Jones' splint was applied, passing through the good shoulder as a counter extension. The child was discharged after six weeks. No deformity was present (Fig. 2).

CASE 4.-N. M, s second child of a poor Italian family, sustained a fracture of the right femur. It was brought to the clinic when 7 days old. A splint was applied. The mother was permitted to take the child home on the second day and told to report for massage. Discharged after five weeks entirely cured (Fig. 3).

CASE 5.-L. T.; second child; hard labor; placenta praevia; born in the hospital. The case was referred to me by Dr. Telfair, and I saw the child one hour after its birth. There was a fracture of right femur and a fracture of the right humerus. Splints were applied immediately. For the upper extremity the ring passed through the same shoulder. Massage was begun two weeks later. There was only one complication. The ring of the femur splint which we happened to have on hand was a little too wide so that it pressed on the left femur causing a slight curvature. That was corrected with massage (Figs. 4,5 and 6 ).

CASE 6.-L. M. C.; born at the hospital with a fractured right humerus. There was no overlapping of fragments. A splint was applied. The patient was discharged cured after four weeks (Figs. 7 and 8 ). 
COMMENT

Of these six cases, three had hospital care, and the splints were watched carefully. The remaining three cases occurred in very poor families where extra care could not be given, still the results were the same. The patients in the hospital gave us the opportunity to observe the methods used carefully, while in the cases of the patients treated at home, the practicability of the method was demonstrated. As a matter of fact, it was proved that it is both safe and convenient to use the splint outside of the hospital. The patients could be dressed easily and carried to and from the dispensary. They could be kept cleaner than under the old methods.

\section{CONCLUSIONS}

1. The Thomas Jones splint can be used with safety in cases of birth fractures affecting the femur or the humerus.

2. It allows easier transportation, permits cleansing of the children and saves a great deal of watching.

3. It permits early massage.

4. The deformity is easily controlled.

5. The union probably occurs earlier on account of the ability of the infant to use the limb, to elevate it together with the brace.

I wish to express my thanks to Dr. Nicoll, the director of the first division, under whose auspices the observations and treatments were made, and to Dr. $\mathrm{J}$. Telfair, the attending obstetrician of the hospital for referring the cases so early. I also wish to express my sincerest thanks to Dr. I. J. Landsman, the radiographer of the hospital, for his roentgenograms.

529 Courtland Avenue. 